\title{
Analysis of histone deposition on specific DNA elements in living mammalian cells
}

\author{
Laetitia Daury and Didier Trouche \\ Laboratoire de Biologie Moléculaire Eucaryote, NRS UMR 5099, Ligue Nationale \\ Contre le Cancer, Toulouse, France
}

BioTechniques 35:326-332 (August 2003)

DNA replication and repair involve the deposition of newly synthesized histones. After their deposition on a specific locus, histones are extensively modified on their protruding $N$-terminal tails to restore the histone code corresponding to this locus. These processes require a complex machinery of histone chaperones and histone-modifying enzymes. The precise kinetics of these events and the identity of the factors involved are still not clear. Here we present a technique based on transient expression of tagged histones followed by chromatin immunoprecipitation that allows the detection of newly synthesized histone deposition on specific DNA elements in mammalian cells at high resolution. This strategy could have important applications in chromatin dynamic studies.

\section{INTRODUCTION}

In eukaryotic cells, nuclear DNA is packaged in chromatin. Chromatin is a highly dynamic structure that interferes with all processes requiring access to the DNA double helix such as transcription, replication, or DNA repair. The basic unit of chromatin, the nucleosome, consists of an octamer of four histones, $\mathrm{H} 3, \mathrm{H} 4, \mathrm{H} 2 \mathrm{~A}$, and H2B. During the process of DNA replication, nucleosomes that were previously present on the replicating DNA are transferred onto one of the newly synthesized doublestranded DNA (dsDNA). Nucleosome formation on the other strand relies on the de novo assembly from newly synthesized histones. Newly synthesized histones are likely to be brought in the vicinity of the replication fork and then assembled in nucleosomes on newly replicated DNA by chromatin assembly factors, such as CAF1 (1). Histonemodifying enzymes are also thought to be critical in this process. Indeed, the epigenetic marks that specify specific functional domains have to be restored on the new nucleosomes (2).

There is also some evidence for histone deposition independently of DNA replication. For example, the DNA synthesis that occurs during some DNA repair processes is also linked to nucleo- some assembly (3). Moreover, histone $\mathrm{H} 2 \mathrm{~A} / \mathrm{H} 2 \mathrm{~B}$ dimers have been proposed to be removed by RNA polymerase II during transcription (4). In addition, replication-independent deposition of a histone $\mathrm{H} 3$ variant has also been reported in differentiated cells (5) or during gene activation in Drosophila (6).

The development of DNA replication or DNA repair in vitro systems allowed the characterization of many histone chaperones or chromatin assembly factors that can accomplish histone deposition (3,7-9). However, the identity of the proteins performing these functions in higher eukaryotic cells is still unclear. Histone deposition in living cells was mainly investigated using green fluorescent protein (GFP)tagged exogenous histones $(6,10)$. A major limitation of these studies is its poor resolution. In addition, since between 20 and 30 GFP molecules are usually needed to detect a fluorescent signal, only loci where massive histone deposition occurs can be analyzed.

Here we present a method that allows for the study of the deposition of newly synthesized histones on specific DNA sequences in mammalian cells. We transiently expressed in mammalian cells a tagged histone H3. The presence of the tag allowed us to distinguish exogenous histones from previously as- sembled histones. This tagged histone was efficiently incorporated into chromatin. Moreover, we were able to detect deposition of this exogenous histone on specific DNA sequences by chromatin immunoprecipitation. Our technique thus allows for the study of histone deposition with the resolution of chromatin immunoprecipitation (a few hundred base pairs). In addition, the deposition of one exogenous histone could be theoretically sufficient to detect a signal. By the use of synchronized cells, this method could allow the study of the precise kinetics of histone deposition on specific sites. By coupling it with RNA interference, it should be possible to characterize the factors that are important for this process. Moreover, a possible development of this method could allow one to monitor the establishment of the epigenetic marks on newly deposited nucleosomes.

\section{MATERIALS AND METHODS}

\section{Cell Culture and Transfection}

NIH-3T3 cells were maintained in Dulbecco's modified Eagle's medium (DMEM; Invitrogen, Carlsbad, CA, USA) supplemented with $10 \%$ fetal calf serum (FCS), antibiotics, and 1\% sodium pyruvate. Cells were transfected using TransFast ${ }^{\mathrm{TM}}$ (Promega, Madison, WI, USA), as recommended by the manufacturer. Twenty-four hours after transfection, the culture medium was changed, and cells were harvested $24 \mathrm{~h}$ later.

\section{Plasmids}

pBos H3-HA and pBos HA empty vectors were constructed by replacing the GFP tag from pBos H3-N-GFP (a kind gift from Dr. P. Cook, Sir William Dunn School of Pathology, Oxford, UK) (11) by a double hemagluttinin (HA) tag in BamHI and NotI. pBos H3.3-HA was constructed by replacing the H3 sequence from pBos H3-HA with that of the $\mathrm{H} 3.3$ variant (a kind gift from Dr. I. di Liegro, Facolta' di Medicina e Chirurgia, Palermo, Italy). In pBos vectors, tagged histones encoding sequences are driven by the elongation factor (EF) $1 \alpha$ promoter. pGEMT- 
DHFR and pGEMT-GAPDH were kindly provided by Dr. A. Harel-Bellan (Institut André Lwoff, Villejuif, France) (12). Details of constructions are available upon request.

\section{Western Blot Analysis and Immunofluorescence}

Western blot analysis was performed using standard procedures.

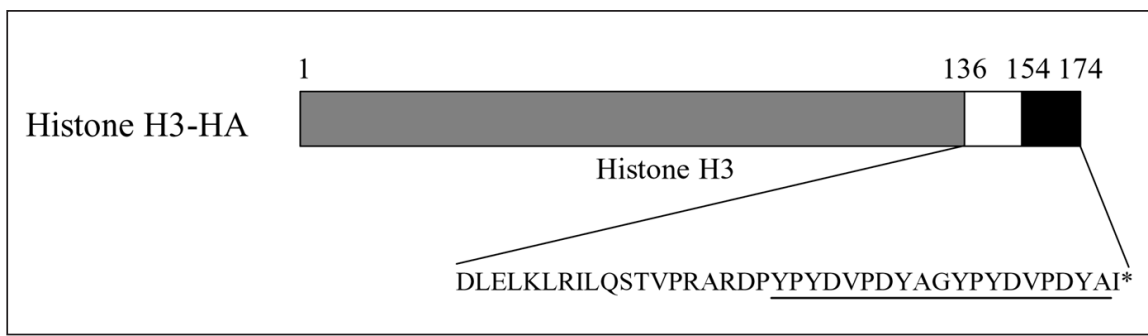

Figure 1. Representation of the tagged histone $\mathbf{H 3}$ used in this study. Human histone $\mathrm{H} 3$ (gray box) (or H3.3 variant) was tagged at its $\mathrm{C}$ terminus using two hemagluttinin (HA) tags (black boxes). Note the presence of a 19-amino acid spacer (empty box) between the histone sequence and the tags. The amino acids that have been added to the histone H3 coding sequence are indicated. The double HA tag is underlined.

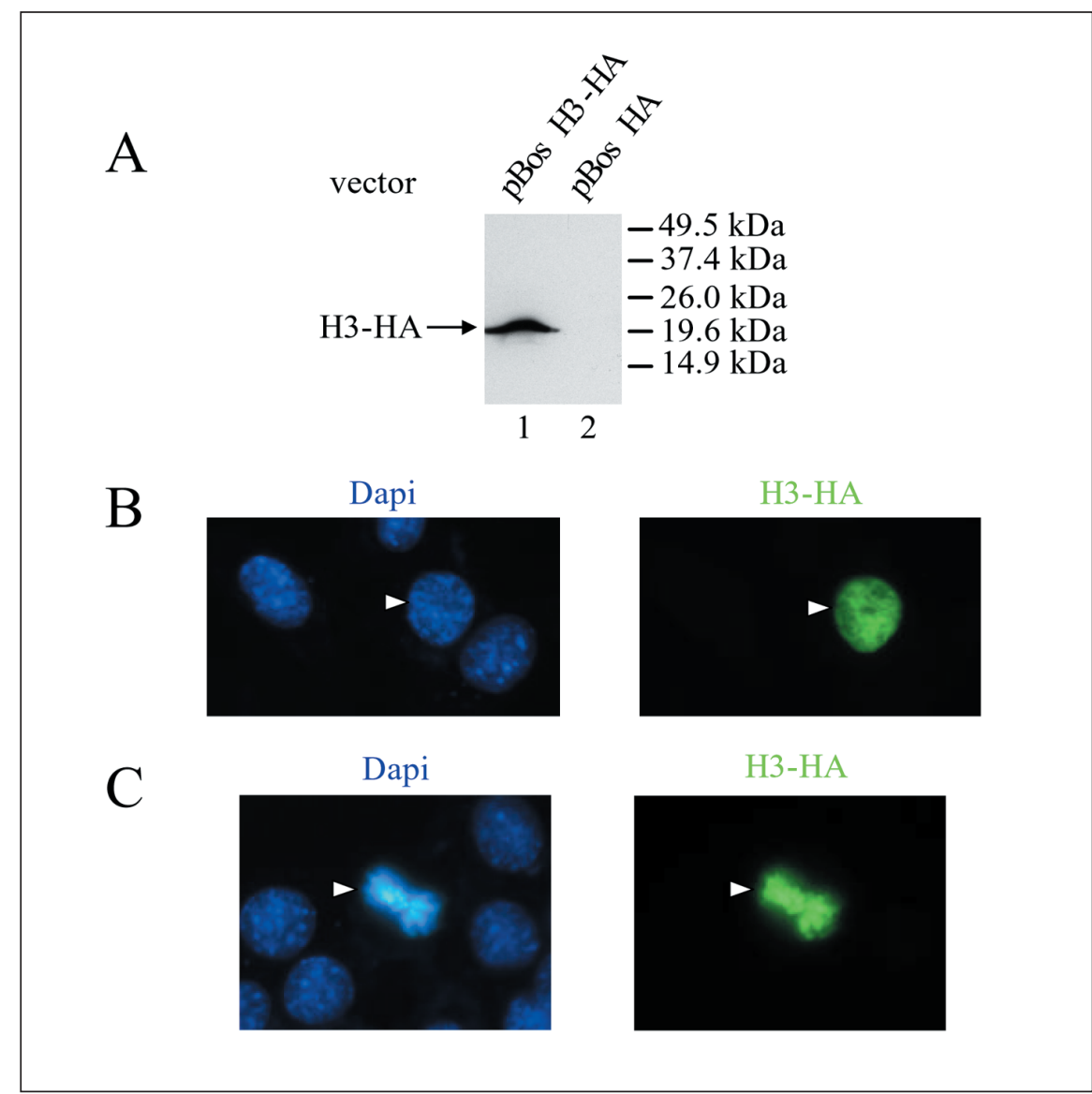

Figure 2. The exogenous hemagluttinin (HA)-tagged histone is incorporated into chromatin. (A) NIH-3T3 cells $(100,000)$ were transiently transfected as indicated in the Materials and Methods section using $1 \mu \mathrm{g}$ pBos H3.3-HA (lane 1) or empty pBos HA vector (lane 2). Forty-eight hours after transfection, cells were directly boiled in Laemmli sample buffer. Extracts were then subjected to an anti-HA Western blot. (B) NIH-3T3 cells $(50,000)$ were transiently transfected using $1 \mu \mathrm{g}$ pBos H3.3-HA. Fortyeight hours after transfection, cells were fixed, permeabilized, and stained with DAPI (left) and by immunofluorescence using the anti-HA antibody (right). The arrow points towards transfected cells. (C) Same as in panel B, except that a mitotic transfected cell is shown.

Briefly, NIH-3T3 cells $(100,000$ cells in $3.5-\mathrm{cm}$ dishes) were transiently transfected using $1 \mu \mathrm{g}$ pBos H3.3-HA or pBos HA empty vector. Forty-eight hours after transfection, cells were directly boiled in Laemmli sample buffer. Lysates were then subjected to Western blot analysis with the anti-HA (16B12) antibody (BAbCO).

For immunofluorescence, NIH-3T3 cells $(50,000$ cells in $3.5-\mathrm{cm}$ dishes $)$ were transiently transfected with $1 \mu \mathrm{g}$ pBos H3.3-HA. Forty-eight hours after transfection, cells were fixed for 15 min with phosphate-buffered saline (PBS) $/ 4 \%$ formaldehyde, permeabilized for $15 \mathrm{~min}$ with $\mathrm{PBS} / 0.5 \%$ Triton ${ }^{\circledR}$ X-100, blocked for $15 \mathrm{~min}$ with $\mathrm{PBS} / 1 \%$ bovine serum albumin (BSA), and stained in PBS $/ 1 \%$ BSA with DAPI and with the anti-HA antibody (16B12, BabCO, used 1/1000 for $1 \mathrm{~h}$ ) and a secondary fluorescein-conjugated antibody.

\section{Chromatin Immunoprecipitations}

Chromatin immunoprecipitations were performed essentially as described previously (12). Briefly, four dishes of NIH-3T3 cells at a density of $1 \times 10^{6}$ cells/10-cm dish were transiently transfected using $30 \mu \mathrm{g}$ pBos H3.3-HA, pBos H3-HA, or pBos HA empty vector/dish. Following formaldehyde cross-linking in living cells, chromatin was purified and subjected to sonication to obtain a mean length of DNA fragments of about $800 \mathrm{bp}$. Sonicated chromatin was then divided in two samples and immunoprecipitated either with $20 \mu \mathrm{L}$ anti-HA (16B12) antibody (BAbCO) or without antibody as a control. After reversion of cross-links and DNA recovery, samples were analyzed by quantitative PCR (ICyclerQ $^{\mathrm{TM}}$; Bio-Rad Laboratories, Hercules, CA, USA), with SYBR $^{\circledR}$ Green I (Sigma, St. Louis, MO, USA) and Platinum Quantitative PCR Supermix-UDG (Invitrogen) to detect DHFR promoter or GAPDH sequences as described previously (12).

The amount of DHFR or GAPDH sequences present in each point was calculated relative to a standard curve obtained using serial dilutions of pGEMT-DHFR or pGEMT-GAPDH vectors, respectively. Four different dilutions of each sample (immunoprecip- 
itates and inputs) were analyzed in parallel. After calculating the mean value for these four dilutions, the efficiency of the immunoprecipitation (in \%) was calculated by dividing the amount of sequences present in the immunoprecipitates by the input material.

\section{RESULTS AND DISCUSSION}

To detect newly synthesized histones, we intended to construct a vector allowing the expression in mammalian cells of tagged exogenous histones. Nucleosomal histone $\mathrm{N}$-terminal tails are extensively subjected to posttranslational modifications. The addition of a tag at the $\mathrm{N}$ terminus could lead to the production of histones that would not be adequately modified, and consequently not efficiently incorporated into chromatin. Histones with a $\mathrm{C}$ terminally fused GFP have been shown to be successfully incorporated into chromatin $(6,10,11,13)$. We thus decided to replace the GFP tag from a histone $\mathrm{H} 3$ used in such a study (11) by a double HA tag to ensure a strong immunoprecipitation efficiency (Figure 1). In addition, the presence of a 19-amino acid spacer between the $\mathrm{C}$ terminus of the histone and the double HA tag favors antibody accessibility. Kimura and Cook (11) showed that histones with a similarly positioned GFP tag were efficiently incorporated into chromatin.

We then transiently transfected an expression vector for these tagged histones into exponentially growing NIH3 T3 cells. Forty-eight hours after transfection, cells were directly boiled into Laemmli sample buffer to ensure an efficient extraction of total cellular proteins. The analysis of HA protein expression by Western blot indicated that the exogenous histone H3 (here the H3.3 variant) was efficiently expressed because a band at the expected molecular weight was detected in cells transfected by the tagged histone $\mathrm{H} 3$ expression vector (Figure 2A, lane 1), but not in cells transfected by the empty vector (Figure 2A, lane 2).

To test whether this exogenous histone was adequately incorporated into chromatin, we performed an immunofluorescence analysis using an anti-HA antibody in transfected cells. Since the cells were cultured for $48 \mathrm{~h}$ after transfection, virtually all cells were passed through S phase. Since the H3.3 variant can undergo replication-dependent deposition (6), the exogenous protein should be incorporated into chromatin. Indeed, we found that the exogenous protein was nuclear (Figure 2B). Moreover, when a mitotic cell was observed, we found that the exogenous protein colocalized with condensed chromosomes (Figure 2C), as expected for a nucleosomal histone. Taken together, these results indicate that the tagged histone was efficiently incorporated into chromatin.
Moreover, they show that the anti-HA antibody could gain access to the chromatin-bound exogenous histone.

This latter result suggests that the presence of exogenous transiently expressed histones on specific DNA elements could be followed up by chromatin immunoprecipitation experiments. To test this possibility, we transiently transfected growing NIH-3T3 cells with the tagged histone $\mathrm{H} 3$ expression vector. Forty-eight hours after transfection, we performed a classical chromatin immunoprecipitation experiment (12). Proteins were cross-linked

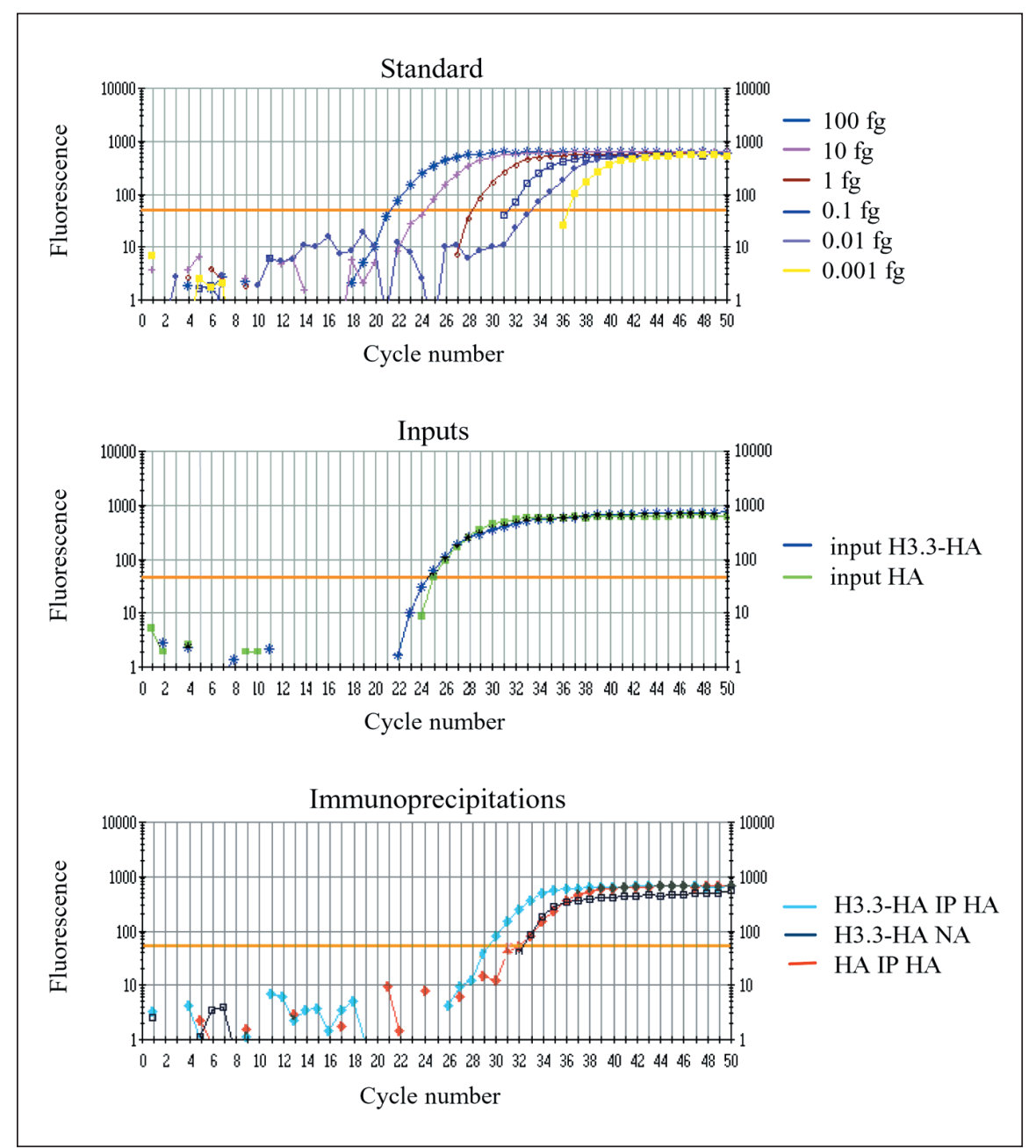

Figure 3. Chromatin immunoprecipitation analysis by real-time PCR of the DHFR promoter. NIH$3 \mathrm{~T} 3$ cells (two $10-\mathrm{cm}$ dishes with $1 \times 10^{6}$ cells each) were transfected with $30 \mu \mathrm{g}$ pBos H3.3-hemagluttinin (HA) or empty pBos HA vector, as indicated. Forty-eight hours after transfection, cells were subjected to a chromatin immunoprecipitation assay using the HA antibody (IP HA) or no antibody (NA). Inputs (middle panel) and immunoprecipitated DNA (bottom panel) were analyzed for the presence of DHFR promoter by real-time PCR. The fluorescence (which is proportional to the amount of PCR product) is plotted against the cycle number. For clarity of results, only one dilution is plotted, but each sample was analyzed in quadruplicate. The top panel shows the result obtained using serial dilutions of a DHFR promoter-containing plasmid. The amount of plasmid used is indicated on the right. The orange lane indicates the threshold used by the software to quantify the starting amount of DNA. 
to DNA by formaldehyde treatment. Chromatin was then fragmented by sonication and immunoprecipitated using the anti-HA antibody. Bound DNA was then analyzed by real-time PCR using primers specific either for the DHFR promoter (Figure 3). We found that, using cells transfected by the tagged histone expression vector, more copies of the DHFR promoter were immunoprecipitated by the anti-HA antibody compared to the no antibody control, as indicated by the fact that the PCR product was detectable earlier (Figure 3, bottom panel). Moreover, immunoprecipitation using the anti-HA antibody from cell extracts transfected by the empty vector led to the recovery of lower amounts of DHFR promoter (Figure 3, bottom panel), although the input DNA was identical (Figure 3, middle panel). Figure 4A shows the quantification of two entirely independent chromatin immunoprecipitation experiments. It indicates that significantly more DHFR sequences were immunoprecipitated by the anti-HA antibody from cell extracts transfected with the H3.3-HA expression vector compared with the control vector. Since the only difference between these two samples is the expression, or lack of expression, of the exogenous tagged histone, this result indicates that our experimental strategy allows the detection of the tagged exogenous histone on the DHFR promoter.

Since these cells have gone through $\mathrm{S}$ phase in which histone deposition is widespread throughout the genome, we

A

DHFR promoter

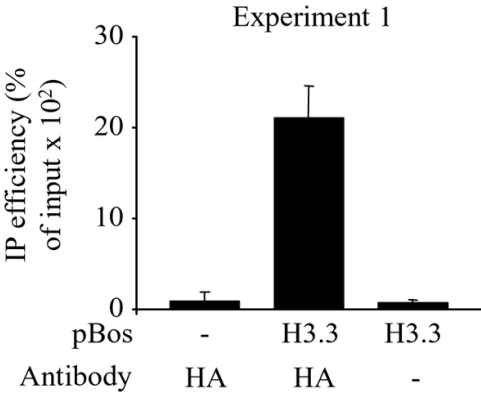

B

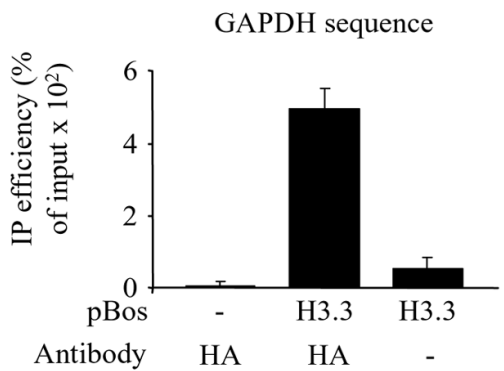

Experiment 2

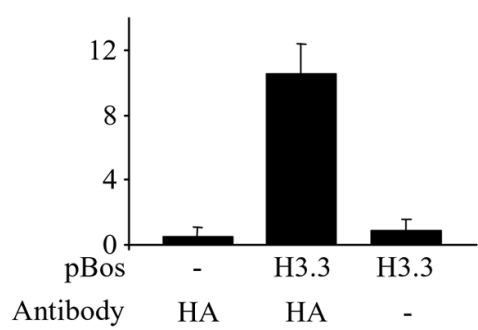

C

DHFR promoter

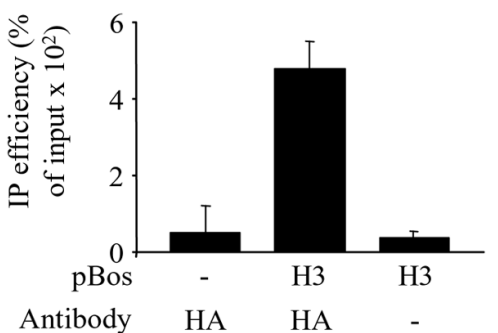

Figure 4. Analysis of tagged histones deposition on specific DNA sequences. (A) Quantification of the results shown in Figure 3. NIH-3T3 cells (two 10 -cm dishes with $1 \times 10^{6}$ cells each) were transfected with $30 \mu \mathrm{g}$ pBos H3.3-hemagluttinin (HA) or empty pBos HA vector as indicated. Forty-eight hours after transfection, cells were subjected to a chromatin immunoprecipitation assay using the HA antibody, or no antibody, as indicated. Immunoprecipitated DNA was analyzed in quadruplicate for the presence of DHFR promoter by real-time PCR. The amount of immunoprecipitated DHFR promoter was calculated relative to the input material. The error bars indicate the standard deviation from the quadruplicate realtime PCR measurements. Two independent chromatin immunoprecipitation experiments are shown. (B) Same as in panel A, except that the presence of GAPDH coding sequences was analyzed by real-time PCR. (C) Same as in panel A, except that NIH-3T3 cells were transfected using pBos H3-HA. 
expect similar histone deposition on any DNA sequence. Accordingly, we found that the GAPDH sequence was also specifically immunoprecipitated by the anti-HA antibody from H3.3HA-expressing cells (Figure 4B). We reproducibly found that the amount of GAPDH sequences immunoprecipitated by the antibody was about 2 -fold lower than DHFR promoter-derived sequences. This may be due to an intrinsic difference between the two sequences. Alternatively, since the efficiency of the deposition of our exogenous histone is likely to depend on the ratio between exogenous histone and endogenous histone, it could vary during $\mathrm{S}$ phase (if the expression of endogenous or exogenous histones changes). Consequently, slight differences in the presence of exogenous histones may exist between early-replicating and latereplicating DNA sequences.

Finally, we found that our technique is not restricted to the study of the H3.3 variant deposition. Immunoprecipitation by an anti-HA antibody using extracts of cells transfected with an expression vector for HA-tagged histone $\mathrm{H3}$, whose deposition is strictly replicationdependent, led to a similar enrichment of the DHFR promoter (Figure 4C).

Taken together, these results indicate that the incorporation of tagged exogenous histones could be detected by chromatin immunoprecipitation. Thus, this method allows the analysis of histone deposition on specific DNA elements.

Strikingly, only a small fraction of input DNA was immunoprecipitated by the anti-HA antibody $(0.05 \%-0.2 \%)$. Of course, this could be due to a low efficiency of tagged histone deposition. However, it has to be noted that we did not sort transfected cells from nontransfected cells in our experiments. Since in our experiments transfection efficiency varied from $2 \%$ to $5 \%$ (data not shown), the immunoprecipitation efficiency from transfected cells raised to around 5\%. Moreover, we found that exogenous histones were expressed at far lower levels than endogenous histones (data not shown), as already shown using pBos-based expression vectors (11). Thus, most nucleosomes would not contain any exogenous histone. Taken together, these data indicate that the relative efficiency of chro- matin immunoprecipitation could certainly be greatly improved by optimizing transfection conditions and/or by eliminating nontransfected cells.

Deposition of exogenous histones in higher eukaryotic cells has already been studied using GFP-tagged histones $(6,10)$. This strategy allowed the study of genome-wide histone deposition. Moreover, it was recently used to investigate histone deposition on the rDNA locus (6). However, the great limitation of this technique is its poor resolution (limited by the resolution of fluorescence microscopy). The technique we present here will allow similar studies at a far better resolution. In chromatin immunoprecipitation experiments, the resolution is given by the average size of DNA fragments used in the immunoprecipitation (in our case, between 0.5 and $1 \mathrm{~kb}$ ). This type of resolution could, for example, allow the study of histone deposition on a specific promoter. In addition, deposition of as few as one histone could be analyzed by our technique, whereas 20-30 molecules are usually required to detect a GFP fluorescent signal.

Our method allows the analysis of histone deposition on specific DNA elements in mammalian cells. It relies on transient transfection, which might be an advantage compared to raising stable cell lines with an inducible expression vector. Indeed, in this latter case, promoter leakage could result in high levels of background immunoprecipitation resulting from incorporation of tagged histones at previous cell cycles. In addition, transient transfection allows the possibility of rapidly testing many different histone mutants.

Our method could also prove to be useful in studies of the kinetics and mechanisms of histone deposition. That our transiently expressed tagged histones are deposited by the normal machinery is indicated by the fact that we do not detect deposition of the histone $\mathrm{H} 3$ variant (that can only undergo replication-dependent deposition) (6) using nonproliferating cells (our unpublished results). The precise kinetics of replication-dependent histone deposition could be analyzed using synchronized cells. In addition, the kinetics of replication-independent histone deposition could be studied in the presence of replication-in- hibiting drugs (such as aphidicolin). Furthermore, the coupling of our method with RNA interference against given factors could allow one to gain insights into the mechanisms of histone deposition on specific elements in mammalian cells. Such information was previously only accessible in yeast models.

Also, a possible development of our technique could allow the analysis of posttranslational modifications of newly deposited histones. Indeed, newly synthesized histones have some specific marks (e.g., histone H4 is acetylated on $\mathrm{K} 5$ and K12). When these histones are incorporated in specific chromatin domains, these marks are removed and replaced by the specific marks of these domains ("epigenetic" marks) (2). The precise kinetics and factors involved in these processes are not known. Successive immunoprecipitations using first the anti-HA antibody then antibodies specific for the various modified histones could allow the determination of the evolution of newly synthesized histone modifications within specific domains. Again, the coupling of these experiments with RNA interference could lead to the characterization of the histone-modifying enzymes involved in these processes.

\section{ACKNOWLEDGMENTS}

The authors wished to thank Drs. P.R. Cook and I. di Liegro for plasmids, Dr. E. Nicolas for helpful discussions, and Dr. A. Harel-Bellan for help with the chromatin immunoprecipitation and real-time PCR experiments. L.D. is supported by a fellowship from the "Ligue Nationale Contre le Cancer". This work was supported by a grant from the "Ligue Nationale Contre le Cancer" to D.T., as an "équipe labellisée".

\section{REFERENCES}

1.Mello, J.A. and G. Almouzni. 2001. The ins and outs of nucleosome assembly. Curr. Opin. Genet. Dev. 11:136-141.

2.Jenuwein, T. and C.D. Allis. 2001. Translating the histone code. Science 293:1074-1080.

3.Gaillard, P.H., E.M. Martini, P.D. Kaufman, B. Stillman, E. Moustacchi, and G. Almouzni. 1996. Chromatin assembly coupled to DNA repair: a new role for chromatin assembly factor I. Cell 86:887-896. 
4.Kireeva, M.L., W. Walter, V. Tchernajenko, V. Bondarenko, M. Kashlev, and V.M. Studitsky. 2002. Nucleosome remodeling induced by RNA polymerase II: loss of the H2A/H2B dimer during transcription. Mol. Cell 9:541-552.

5.Lennox, R.W. and L.H. Cohen. 1988. The production of tissue-specific histone complements during development. Biochem. Cell Biol. 66:636-649.

6.Ahmad, K. and S. Henikoff. 2002. The histone variant H3.3 marks active chromatin by replication- independent nucleosome assembly. Mol. Cell 9:1191-1200.

7.Smith, S. and B. Stillman. 1989. Purification and characterization of CAF-I, a human cell factor required for chromatin assembly during DNA replication in vitro. Cell 58:15-25.

8.Verreault, A., P.D. Kaufman, R. Kobayashi, and B. Stillman. 1996. Nucleosome assembly by a complex of CAF-1 and acetylated histones $\mathrm{H} 3 / \mathrm{H} 4$. Cell 87:95-104.

9.Ito, T., M.E. Levenstein, D.V. Fyodorov, A.K. Kutach, R. Kobayashi, and J.T. Kadonaga. 1999. ACF consists of two subunits, Acf1 and ISWI, that function cooperatively in the ATP-dependent catalysis of chromatin assembly. Genes Dev. 13:1529-1539.

10.Henikoff, S., K. Ahmad, J.S. Platero, and B. van Steensel. 2000. Heterochromatic deposition of centromeric histone H3-like proteins. Proc. Natl. Acad. Sci. USA 97:716-721.

11.Kimura, H. and P.R. Cook. 2001. Kinetics of core histones in living human cells: little exchange of $\mathrm{H} 3$ and $\mathrm{H} 4$ and some rapid exchange of H2B. J. Cell Biol. 153:1341-1353.

12.Ferreira, R., I. Naguibneva, M. Mathieu, S. Ait-Si-Ali, P. Robin, L.L. Pritchard, and A. Harel-Bellan. 2001. Cell cycle-dependent recruitment of HDAC-1 correlates with deacetylation of histone $\mathrm{H} 4$ on an Rb-E2F target promoter. EMBO Rep. 2:794-799.

13.Kanda, T., K.F. Sullivan, and G.M. Wahl. 1998. Histone-GFP fusion protein enables sensitive analysis of chromosome dynamics in living mammalian cells. Curr. Biol. 8:377385 .

Received 13 March 2003; accepted 7 May 2003.

Address correspondence to Didier Trouche, CNRS UMR 5099, Laboratoire de Biologie Moléculaire Eucaryote, 118, Route de Narbonne, 31062 Toulouse Cedex, France. e-mail:trouche@ibcg.biotoul.fr

\title{
Aberrant cryptic responsiveness of the $\mathrm{pCAT}^{\circledR} 3$ - and pGL3-promoter reporter vectors
}

\author{
G. Giannakis1,2, S.R. Edmondson'1, J.M. Favaloro33, J.D. Zajac ${ }^{1,2}$, \\ and K.J. Greenland ${ }^{1}$ \\ ${ }^{1}$ Murdoch Childrens Research Institute, ${ }^{2}$ Austin and Repatriation Medical Center, \\ ${ }^{3}$ Royal Melbourne Hospital, University of Melbourne, Victoria, Australia
}

BioTechniques 35:332-339 (August 2003)

Transfection analyses are an informative method to assess the activity of specific promoter or enhancer elements in mammalian cells. Commercially available reporter vectors can be extremely useful investigative tools for such studies. This study reports that the $p C A T^{\circledR} 3$-and pGL3-promoter vectors display cryptic responsiveness to androgens when they contain a DNA insert, while the empty vector, a commonly used negative control, is nonresponsive. Our studies initially aimed to characterize novel androgen-responsive DNA sequences in human genomic DNA through transactivational analyses. An isolated DNA fragment, designated ARC-3, contained three putative androgen response element "half-sites" and was androgen-responsive when cloned into the pCAT3-promoter vector. While we originally believed this to be a novel enhancer element, subsequent analyses of this clone revealed that this vector displays cryptic activity in the presence of an androgen. This was confirmed by cloning several unrelated DNA fragments that did not contain any known classic response elements into the pCAT3-promoter vector, all of which were found to be responsive. The empty vector (negative control) was again nonresponsive. The ARC-3 DNA fragment was also weakly responsive to stimulation when cloned into the pGL3-promotor vector, which is identical to the pCAT3-promoter vector, with the exception of an intron located 5' of the chloramphenicol acetyltransferase gene, and the reporter genes. This work demonstrates that both the pCAT3- and pGL3-promoter vectors are inappropriate to assess androgen-responsive enhancers and emphasizes the importance of the careful selection of reporter vectors and controls when conducting transactivational analysis.

\section{INTRODUCTION}

Transfection analyses in mammalian cells are a standard method to assess the transactivational abilities of gene promoters and enhancer elements. There are several commercially available reporter vectors for analyzing the activity of DNA fragments. Such activity is measured through the expression of downstream reporter genes such as chloramphenicol acetyltransferase (cat) or luciferase $(l u c)$. Our studies are focused on the analysis of promoters and enhancers regulated by androgens. Transcriptional regulation mediated by the androgen receptor (AR) requires the binding of the ligand-bound receptor complex to DNA sequences, known as hormone response elements (HREs) or androgen response elements (AREs), which are present in or near the promoter regions of responsive genes. Consensus AREs, through which the AR can modulate gene transcription, consist of two inverted nonidentical hexamers that are separated by a three-nucleotide spacer $(1,2)$. Nonconsensus binding sites may consist of different arrangements of these hexamers or "half-sites." We aimed to identify novel AREs using the technique of whole-genome PCR, a method by which genomic DNA sequences are isolated through the binding of a transcription factor (3), in our case, the AR. One of the isolated clones, which was highly responsive to androgens in initial transactivation analysis, was designated ARC-3. Further analysis demonstrated the presence of three putative consensus ARE half-sites $(4,5)$ that we initially believed were responsible for the androgen-responsiveness of ARC3. The original aim of this investigation was to identify which regions of ARC- 